\title{
Studies of the Effects of Administration of ACTH and Adrenal Corticosteroids on Erythrophagocytosis *
}

\author{
Robert M. Greendyke, Edwin M. Bradley, and Scott N. Swisher $\dagger$ \\ With THE TEChNICAL assistance of Norma C. TRabold \\ (From the Departments of Pathology and Medicine, University of Rochester School of \\ Medicine and Dentistry, Rochester, N. Y.)
}

Phagocytosis of effete erythrocytes or red cell fragments by reticuloendothelial cells appears to play some role in normal red cell destruction, although the details of the process are poorly understood. The marked increase in erythrophagocytosis occurring in several types of hemolytic anemia, especially in acquired hemolytic disease and in some examples of transfusion reactions and erythroblastosis fetalis, indicates the possible importance of this process as a mechanism of red cell destruction. The rapid, often dramatic, suppression of erythrocyte destruction in patients with acquired hemolytic disease frequently produced by therapeutic adrenal corticosteroids suggests that these compounds may exert a specific effect on phagocytosis of these abnormal erythrocytes, apart from possible suppression of production of antibody-like proteins. The present study extends preliminary observations (1) on the suppression of the in vitro erythrophagocytic capabilities of peripheral blood leukocytes obtained from patients receiving ACTH or adrenal corticosteroids.

\section{Methods}

The details of the basic experimental procedure employed and a discussion of factors influencing in vitro erythrophagocytosis have been presented previously (2). The basic experimental procedure is as follows: Equal volumes of a $3 \%$ suspension in $0.9 \%$ saline of three times washed, fresh erythrocytes from a normal group A donor and a suitable dilution of complement fixing

* Submitted for publication November 16, 1964 ; accepted January 14, 1965.

This study was supported by research grant AM00537-15, National Institute of Arthritis and Metabolic Diseases, and grant FR-44-03, Division of Research Facilities and Resources, National Institutes of Health.

$\dagger$ Address requests for reprints to Dr. Scott N. Swisher, Hematology Unit, University of Rochester School of Medicine and Dentistry, Rochester, N. Y. 14620.
anti-A antibody are combined. After incubation for 30 minutes at $25^{\circ} \mathrm{C}$, the cells are again washed three times in saline and resuspended in $0.9 \%$ saline buffered to $\mathrm{pH}$ 7.3 with one-fifth part isotonic phosphate buffer. One volume of a leukocyte suspension in fresh, autologous heparinized plasma containing 11,000 to 12,000 leukocytes and 20,000 to 30,000 erythrocytes per $\mathrm{mm}^{8}$ is added. The mixture is incubated for 1 hour at $37^{\circ} \mathrm{C}$, gently centrifuged, and the cell sediment smeared and stained with Wright's stain. Five hundred neutrophils and monocytes are examined, and the percentage exhibiting erythrophagocytosis is determined.

Preliminary studies of the effect of adrenal corticosteroids added to leukocyte suspensions in vitro were carried out by these methods. Aqueous solutions of soluble corticosteroids were added to human leukocyte suspensions in fresh heparinized autologous plasma, and the mixture was incubated for varying periods of time at $25^{\circ} \mathrm{C}$. At intervals, samples of this mixture were added to suspensions of human group A erythrocytes previously opsonized. The experiments were then completed as above. $\mathrm{pH}$ values in the supernatant fluid of experimental and control tubes were determined. Complement levels were assayed by adding dilutions of this supernatant fluid to sensitized sheep red cells. The resulting hemolysis was read visually. In some studies, the leukocytes were washed and resuspended in fresh autologous plasma before being added to sensitized erythrocytes. Control studies were carried out to rule out effects of the compounds added on the opsonification portion of the overall procedure.

Experiments were carried out to ascertain the daily and diurnal variation of erythrophagocytic capacity of the leukocytes of a number of donors. These studies were carried out by obtaining serial blood samples from human experimental subjects at intervals; combining leukocyte suspensions in fresh heparinized autologous plasma from these samples with group $A_{1}$ erythrocytes previously sensitized with anti-A, washed, and suspended in buffered saline; and completing the experiments as above. In certain studies, the leukocyte suspensions were prepared in plasma obtained from compatible donors. Because of the critical dependence of the amount of erythrophagocytosis on the degree of opsonification of the erythrocytes in these studies, samples from a single lot of sensitized red cells were used throughout individual experiments. Rigidly quantitative technique was 
employed throughout in preparation and measurement of all cell samples, with adjustment of the leukocyte count of the leukocyte suspensions to comparable values (11,000 to 12,000 per $\mathrm{mm}^{3}$ ) before use.

Experiments involving infusion of ACTH into human volunteers included a sham intravenous infusion of 700 to $800 \mathrm{ml}$ of $5 \%$ detrose in water over a 6- to 8hour period on one day and the intravenous infusion of
$20 \mathrm{U}$ of ACTH in a similar volume of dextrose and water the following day. Small samples of venous blood for determination of leukocyte erythrophagocytic ability were obtained at intervals throughout these infusions, and approximately 3 hours after the start of each infusion a sample of arterial blood was obtained for determination of plasma cortisol levels.

A similar protocol was followed for experiments on

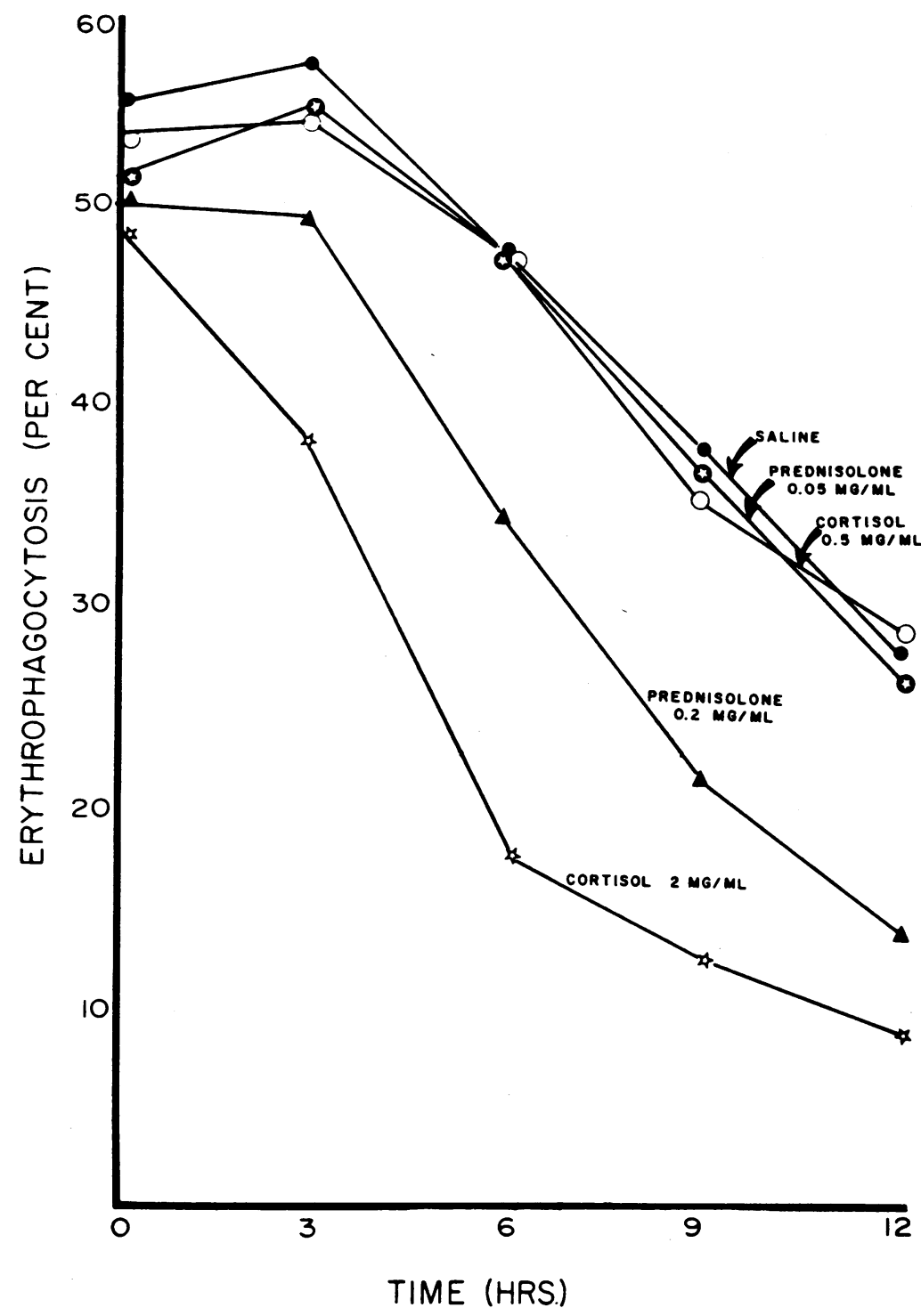

Fig. 1. EFfect ON ERYTHROPHAgocytosis OF INCUBATING LEUKOCYTES IN FRESH HEPARINIZED AUTOLOGOUS PLASMA WITH EQUAL AMOUNTS OF ISOTONIC SALINE SOLUTIONS, PH 7.4, OF CORTISOL (0.5 AND 2 MG PER ML), PREDNISOLONE (0.05 AND 0.2 MG PER ML), OR ISOTONIC SALINE ALONE FOR VARYING PERIODS OF TIME BEFORE COMBINATION WITH OPSONIZED ERYTHROCYTES FOR A STANDARD PERIOD OF PHAGOCYTOSIS. The decline in erythrophagocytic ability of leukocytes aged in plasma plus saline is due, at least in part, to accumulation of inhibitory substances in the suspending plasma (4). 
the effect of intravenously administered cortisol. By means of a constant infusion pump, cortisol (hydrocortisone, alcohol U.S.P.) was infused into an arm vein at a rate of $14 \mu \mathrm{g}$ per minute, or $160 \mu \mathrm{g}$ per minute, for low and high dose levels, respectively. The duration of the infusions was 4 to 6 hours. Aldosterone alcohol was infused in similar fashion at the rate of $0.012 \mu \mathrm{g}$ per minute for 4 hours in a single experiment. In the studies on the effects of prednisone, the drug was administered orally in a single dose of $80 \mathrm{mg}$, and the above general experimental outline was then followed.

Quantitative estimation of the concentrations of plasma cortisol were carried out by the method of Plager, Cushman, and Chase (3). Blood was collected in heparinized syringes. The plasma was separated within 30 minutes and stored frozen until analyzed. Prednisone levels were determined in a similar fashion except that the determination was performed on the eluate of the prednisone area of the paper chromatogram.

\section{Results}

A series of experiments was carried out to determine the effect on erythrophagocytosis of incubating in vitro normal leukocytes suspended in fresh autologous heparinized plasma with varying concentrations of cortisone acetate, cortisol, desoxycorticosterone acetate (DOCA), and prednisolone for varying periods of time before combination with opsonized erythrocytes. Such experiments showed no inhibition by DOCA in concentrations up to $0.5 \mathrm{mg}$ per $\mathrm{ml}$. Inhibition of leukocyte erythrophagocytic ability by the three

TABLE I

Erythrophagocytosis*

\begin{tabular}{clll}
\hline \hline Donor no. & Experiment 1 & Experiment 2 & Experiment 3 \\
\hline & $\%$ & $\%$ & $\%$ \\
1 & 64 & 68 & 64 \\
2 & 82 & 84 & 81 \\
3 & 68 & 72 & 68 \\
4 & & 67 & 70 \\
5 & 25 & 29 & 31 \\
6 & 47 & 41 & 41 \\
7 & 43 & 43 & 46 \\
8 & 53 & 57 & 58 \\
9 & 22 & 20 & 25 \\
10 & 44 & 42 & 46 \\
11 & 56 & 57 & 59 \\
12 & 53 & 55 & 50 \\
Mean and SD & $52.0 \pm 0.7$ & $52.9 \pm 0.7$ & $53.3 \pm 0.7$ \\
\hline
\end{tabular}

* Erythrophagocytic ability of leukocy tes from 12 fasting healthy adult donors of blood group $\mathrm{A}_{1}$ bled simultaneously on three different occasions 5 days apart. The differences between the mean values of the three sets of experiments are not statistically significant. The differences from donor to donor are highly significant $(p<0.001)$. The $\mathrm{SD}$ of a single observation is estimated to be $2.4 \%$. glucocorticoids was not demonstrated until grossly unphysiological quantities of the test compound (of the order of 0.2 to $2.0 \mathrm{mg}$ per $\mathrm{ml}$ ) were added to the incubation medium. These experiments do not lend themselves to tabulation or statistical treatment because of the variability from one to another of degree of erythrocyte opsonification and phagocytic activity of donor leukocytes, resulting in differences in base-line values and rates of inhibition of erythrophagocytosis. The results of a representative experiment demonstrating inhibition of erythrophagocytosis by in vitro incubation of leukocytes suspended in plasma with added prednisolone or cortisol are shown in Figure 1. With the assay employed, no inhibition of complement activity by the test compound as compared with normal saline controls was found in these experiments. A modification of these studies was also carried out; after preincubation of the leukocytes in plasma plus steroid (or normal saline as a control) for intervals of up to 12 hours, the leukocytes were washed twice and resuspended in autologous heparinized plasma before addition of opsonized erythrocytes. Under these conditions, leukocytes preincubated with cortisol $(2 \mathrm{mg}$ per $\mathrm{ml})$ or prednisolone $(0.2 \mathrm{mg}$ per $\mathrm{ml})$ exhibited the same phagocytic ability as did the controls in which saline had been added to the suspending plasma instead of to the test compound.

Before studies on the effects of corticosteroids administered in vivo were begun, a series of preliminary investigations was carried out to determine the variation with time in erythrophagocytic ability of leukocytes from various donors. Fourteen experiments were conducted in which normal volunteers (nine subjects) or patients with moderately severe chronic adrenal cortical insufficiency (two subjects) were bled at 1- to 4-hour intervals between 9:00 a.m. and midnight, while at rest, either with or without the administration of an intravenous infusion of glucose in water during this time. No diurnal variation was demonstrated in leukocyte erythrophagocytic ability in any subject when fresh leukocytes were employed.

Another study was carried out to determine the variability of leukocyte erythrophagocytic ability among a group of healthy adults both from the viewpoint of donor to donor variability at a given time of day and variability in individual 
donors studied at the same time on different days. Twelve healthy adult donors, seven females and five males, aged 20 to 65 years, all of erythrocyte group $A_{1}$ and all with normal total and differential leukocyte counts, were simultaneously bled after an overnight fast on each of three occasions separated by 5 days each. Leukocyte suspensions were prepared from each sample, adjusted to a uniform concentration, and combined with samples of opsonized $A_{1}$ erythrocytes from a single donor. The variability in erythrophagocytic ability from donor to donor and the uniformity of this ability in leukocytes obtained from single donors on different days are demonstrated by the results shown in Table I. The complement titers of all donors on all three occasions were the same.

To ascertain whether the differences observed in the erythrophagocytic ability of leukocytes from various donors were due to serum factors or to primary differences among the leukocytes, leukocyte suspensions were prepared as above from 10 of the 12 healthy adult donors previously mentioned. The leukocytes were washed twice in saline, and samples of each were resuspended in $a)$ fresh heparinized autologous plasma, $b$ ) fresh heparinized plasma from Donor 2, whose leuko-

TABLE II

Erythrophagocytosis*

\begin{tabular}{|c|c|c|c|}
\hline Donor no. & $\begin{array}{c}\text { Leukocytes } \\
\text { in autol- } \\
\text { ogous plasma }\end{array}$ & $\begin{array}{c}\text { Leukocytes } \\
\text { in plasma } \\
\text { from Donor } 2\end{array}$ & $\begin{array}{l}\text { Leukocytes } \\
\text { in plasma } \\
\text { from Donor } 5\end{array}$ \\
\hline & $\%$ & $\%$ & $\%$ \\
\hline 1 & 68 & 74 & 42 \\
\hline 2 & 81 & 84 & 59 \\
\hline 3 & 69 & 79 & 63 \\
\hline 4 & 70 & 74 & 52 \\
\hline 5 & 30 & 57 & 33 \\
\hline 7 & 46 & 62 & 33 \\
\hline 8 & 60 & 69 & 52 \\
\hline 9 & 29 & 67 & 30 \\
\hline 11 & 60 & 68 & 43 \\
\hline 12 & 49 & 55 & 30 \\
\hline Mean and SD & $56.2 \pm 2.1$ & $68.9 \pm 2.1$ & $43.7 \pm 2.1$ \\
\hline
\end{tabular}

* Erythrophagocytic ability of leukocytes from ten fasting healthy adult donors of blood group $A_{1}$ bled simultaneously. The leukocytes were washed and resuspended in autologous plasma, and in plasma from donors showing high (Donor 2) and low (Donor 5) erythrophagocytic ability of their leukocytes when suspended in their respective autologous plasmas. Samples of opsonized erythrocytes from a single donor were employed in all cases.

The results in the second column are significantly higher $(t=4.29$ with 18 degree of freedom, $\mathrm{p}<0.001)$ and in the third column are significantly lower $(t=4.23$ with 18 degrees of freedom, $\mathrm{p}<0.001$ ) than in the first column. The SD of a single observation is estimated at $6.6 \%$.

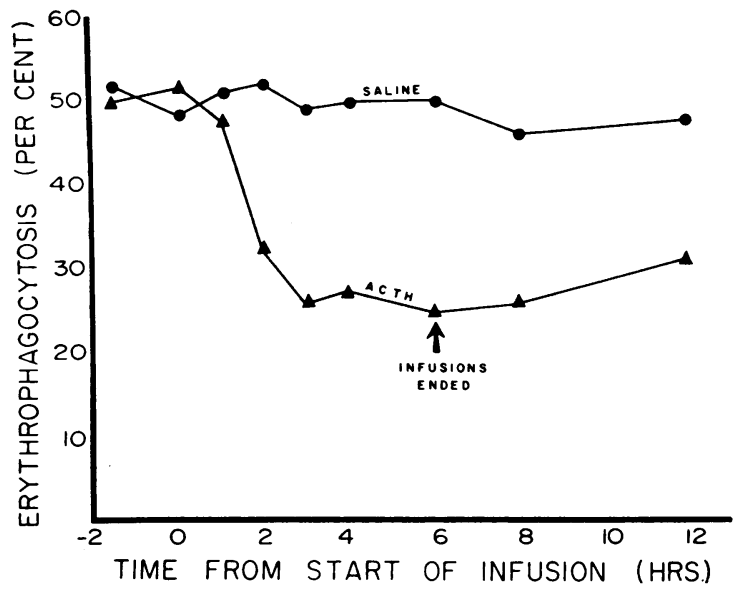

Fig. 2. EFFECT ON ERYThRophagocytosis OF INTRAVENOUS INFUSION OF SALINE OR $20 \mathrm{U}$ OF ACTH OVER A 6-HOUR PERIOD TO NORMAL LEUKOCYTE DONOR.

cyte suspensions in autologous plasma had previously shown very active erythrophagocytic ability, and c) fresh heparinized plasma from Donor 5, whose leukocyte suspensions in autologous plasma had shown very low erythrophagocytic ability. Samples of opsonized erythrocytes from a single donor were added and the experiments completed. The results of this study are shown in Table II ; it will be seen that enhancement of erythrophagocytic ability, averaging $38 \%$, was obtained by incubating various donors' leukocytes in plasma from Donor 2, and depression of an average of $23 \%$ was effected by plasma from Donor 5 . Again, no differences in complement activity in the plasma samples from any of the donors were demonstrated by the method utilized.

In a series of experiments on the effect of in vivo administration of ACTH, a total of eight studies was carried out on five normal subjects, and two studies were performed using two patients with well documented chronic adrenal cortical insufficiency. The typical alteration in erythrophagocytic ability of leukocytes from a normal subject during intravenous $\mathrm{ACTH}$ infusion and the lack of this effect during infusion of glucose in water are shown in Figure 2. The plasma cortisol values for this subject 3 hours after the start of the ACTH infusion were $34.5 \mu \mathrm{g}$ per $100 \mathrm{ml}$. Normal plasma cortisol levels without adrenal stimulation by this method are less than $10 \mu \mathrm{g}$ per $100 \mathrm{ml}$. Similar results were obtained in each 
TABLE III

Effect on erythrophagocytic ability of ACTH infusion in five normal subjects on eight occasions*

\begin{tabular}{ccc}
\hline \hline & $\begin{array}{c}\text { Maximal de- } \\
\text { crease in } \\
\text { erythrophago- } \\
\text { cytosis rela- } \\
\text { tive to ini- } \\
\text { tial value }\end{array}$ & $\begin{array}{c}\text { Plasma } \\
\text { cortisol } \\
\text { concentration }\end{array}$ \\
\hline Patient & $\%$ & $\mu g / 100 \mathrm{ml}$ \\
W. H. & -55 & 34.5 \\
N. H. & -50 & 79.0 \\
N. H. & -47 & 53.2 \\
W. S. & -28 & 98.8 \\
W. S. $\dagger$ & -26 & 17.2 \\
H. B. & -50 & 47.9 \\
H. K. & -39 & 52.3 \\
H. K. & -48 & \\
\hline
\end{tabular}

* The maximal effect was noted approximately 3 hours after the infusion was begun. Plasma cortisol levels were determined at this time.

$\dagger$ Patients N. H. and W. S. were restudied after 4 and 2 weeks, respectively, of intensive estrogen therapy. The rise in plasma cortisol levels is thought to be present chiefly in the protein-bound fraction and not available to influence leukocyte function as much as would a comparable rise in unbound cortisol (5).

of the seven other studies of this type that were conducted. Maximal depression of erythrophagocytic ability was present within 3 hours after the start of the ACTH infusion, the drop averaging $43 \%$ of the initial values. The percentage fall in erythrophagocytosis between the start of the $\mathrm{ACTH}$ infusion and the time of maximal depression plus the concentrations of plasma cortisol 3 hours after the start of the infusion in these stud-

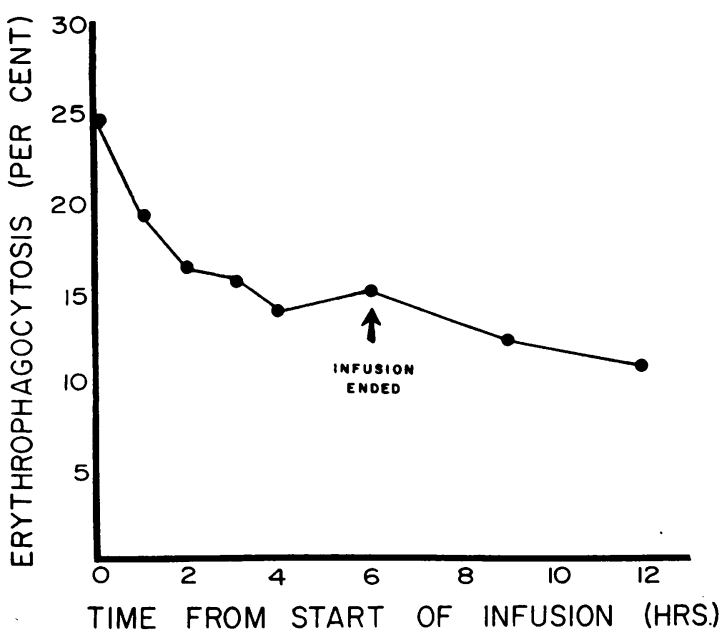

Fig. 3. EFFECT ON ERYTHROPHAGOCYTOSIS OF INTRAVENOUS INFUSION OF 55 MG OF CORTISOL OVER A 6-HOUR PERIOD TO A NORMAL LEUKOCYTE DONOR.

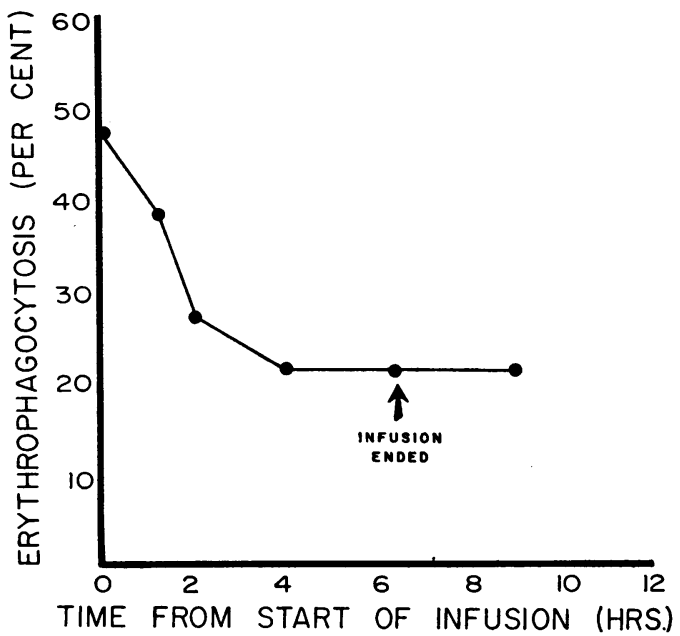

Fig. 4. EFFect ON ERYTHROPHAGOCYTOSIS OF INTRAVENOUS INFUSION OF 65 MG OF CORTISOL OVER A $6 \frac{1}{2}$ HOUR PERIOD TO A PATIENT WITH CHRONIC ADRENAL CORTICAL INSUFFICIENCY.

ies are listed in Table III. No significant variation in leukocyte type or maturity was demonstrated in the course of these studies to explain the results observed.

Similar studies in two patients with chronic adrenal cortical insufficiency normally maintained on 17.5 and $25 \mathrm{mg}$ of cortisone per day, respectively, disclosed that ACTH infusion exerted no effect on the erythrophagocytic ability of leukocytes obtained from these subjects, the amount of erythrophagocytosis remaining unchanged throughout the studies. Both patients had received no exogenous adrenal cortical hormone or analog for 24 hours before the test was begun. The plasma cortisol levels of the patient in the experiment shown were $8.2 \mu \mathrm{g}$ per $100 \mathrm{ml} 3$ hours after the ACTH infusion was begun. The erythrophagocytic ability of the leukocytes of each of these two patients was later shown to be depressed normally by intravenously administered cortisol or orally administered prednisone.

Cortisol was administered in a dose of $55 \mathrm{mg}$ intravenously during a period of 6 hours to a normal subject, with the effects on leukocyte erythrophagocytosis shown in Figure 3 . The plasma cortisol level 3 hours after the start of the infusion was $36.4 \mu \mathrm{g}$ per $100 \mathrm{ml}$. Similarly, a patient with chronic adrenal cortical insufficiency normally maintained on $17.5 \mathrm{mg}$ of cortisone daily, 
who had received no medication for 24 hours, was given $65 \mathrm{mg}$ of cortisol intravenously over a $6 \frac{1}{2}-$ hour period, with the results shown in Figure 4. Three hours after the start of the infusion the plasma cortisol level was $65.6 \mu \mathrm{g}$ per $100 \mathrm{ml}$. In both cases, sham infusions on the previous day caused no alteration in erythrophagocytosis. Two similar studies on normal subjects and one study on a patient with chronic adrenal cortical insufficiency in which physiological amounts of cortisol, $16 \mu \mathrm{g}$ per minute, were infused for 6 hours showed no effect on erythrophagocytosis and did not raise the plasma cortisol levels above normal.

Figures 5 and 6 show the effects on the erythrophagocytic ability of peripheral blood leukocytes of oral administration of a single $80-\mathrm{mg}$ dose of prednisone to a normal volunteer (Figure 5 ) and to a patient with chronic adrenal cortical insufficiency (Figure 6). The latter patient was normally maintained on $25 \mathrm{mg}$ of cortisone daily and had received no medication for 24 hours. The plasma prednisone levels in the two studies 3 hours after administration of the drug were 44.6 and $61.0 \mu \mathrm{g}$ per $100 \mathrm{ml}$, respectively.

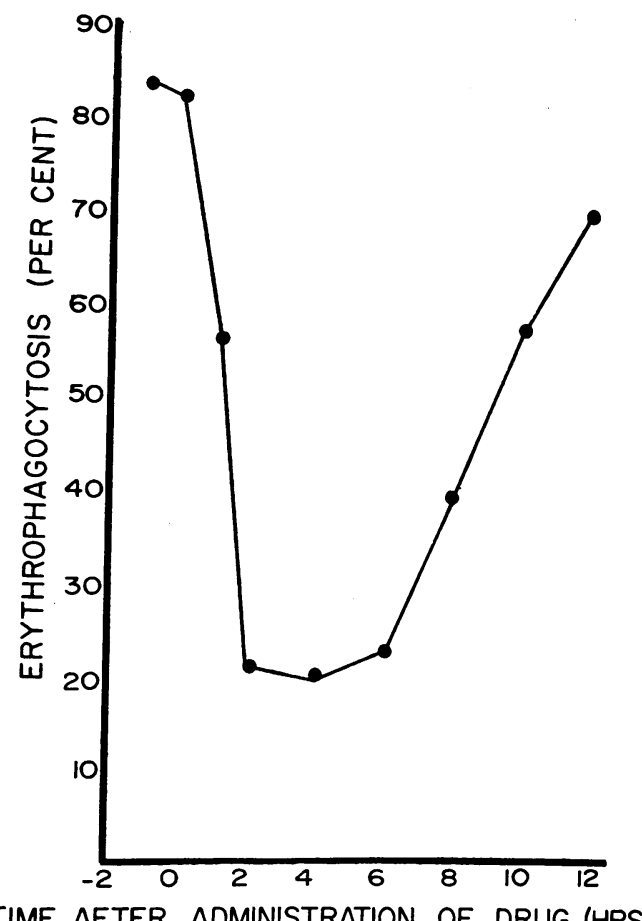

TIME AFTER ADMINISTRATION OF DRUG (HRS)

Fig. 5. EFFECT ON ERYTHROPHAGOCYTOSIS OF ORAL ADMINISTRATION OF A SINGLE 80-MG DOSE OF PREDNISONE TO A NORMAL LEUKOCYTE DONOR.

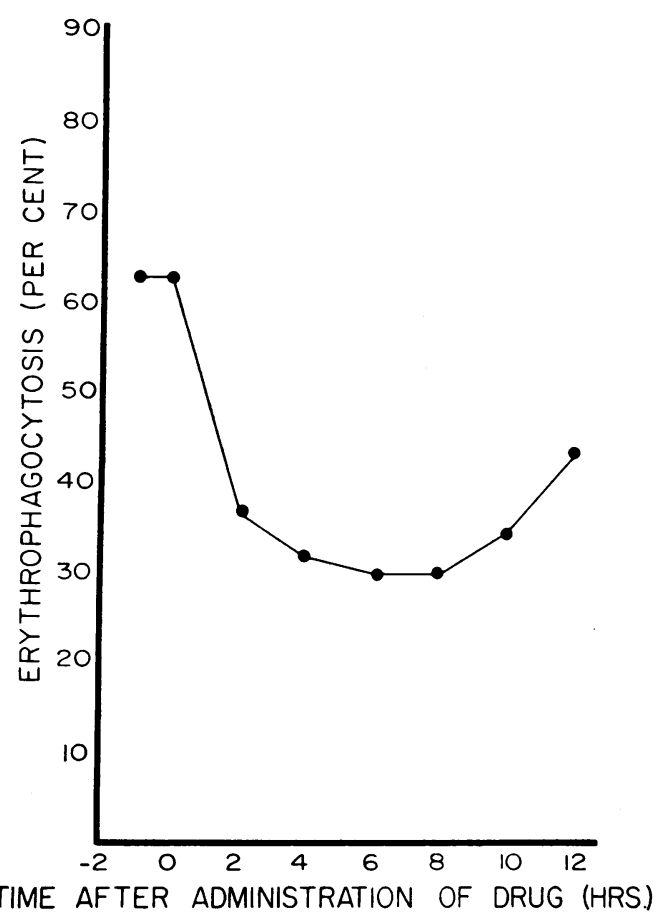

Fig. 6. EFFECT ON ERYTHROPHAGOCYTOSIS OF ORAL ADMINISTRATION OF A SINGLE 80-MG DOSE OF PREDNISONE TO A PATIENT WITH CHRONIC ADRENAL CORTICAL INSUFFICIENCY.

Other experiments were carried out in which leukocytes obtained before the start of an ACTH or "high concentration" cortisol infusion and leukocytes obtained 4 hours after the start of the infusion were washed and samples of each resuspended in both the "0-hour" and "4-hour" plasma. The decreased erythrophagocytic ability found in 4-hour leukocytes suspended in 4-hour plasma was returned to the original higher value by suspension in 0-hour plasma; conversely, 0-hour leukocytes suspended in 4-hour plasma showed inhibition of erythrophagocytosis comparable to that seen in 4-hour leukocytes suspended in 4-hour plasma. Studies of this type during three ACTH infusion tests on normal subjects and one high concentration cortisol infusion in a patient with chronic adrenal cortical insufficiency yielded comparable results.

A single patient with chronic adrenal cortical insufficiency, previously shown to be responsive in erythrophagocytic studies to large amounts of intravenously administered cortisol, was given an intravenous infusion of $2.9 \mathrm{mg}$ of aldosterone 
over a 4-hour period. Serial tests of erythrophagocytic ability of his peripheral blood leukocytes during the infusion and for 3 hours after its termination showed no change.

\section{Discussion}

Considerable attention has been paid by investigators to elucidation of the effects of therapeutic ACTH and adrenal corticosteroids on the process of phagocytosis. Early observations on the deleterious effects of glucocorticoid administration in many infectious diseases led to extensive studies of effects of steroid compounds on the clearance of various intravenously injected materials by the reticuloendothelial system of the intact animal. Inconclusive or contradictory results were obtained in certain of the studies, some of which showed increased and others decreased phagocytic activity by the fixed reticuloendothelial elements (6-11) under the influence of glucocorticoids or ACTH. Similar inconsistent effects of glucocorticoids or ACTH were noted by investigators working with phagocytosis of bacteria by exudate macrophages and blood leukocytes (12-18). All investigators appear to agree on the lack of effect on phagocytosis of corticosteroid compounds lacking glucocorticoid activity. As pointed out by the work of Crabbe (14-16), Reichard, Edelmann, and Gordon $(8,9)$ and Snell (11), most of the discrepancies seem to be accounted for by differences in phagocytic activity toward various types of test materials and by the general pharmacological principle that many compounds that stimulate in low concentrations are inhibitory at higher levels. It should be emphasized that almost none of the previous work in this area has included actual measurement of blood corticosteroid concentrations at the time that phagocytic capabilities were being assessed; the studies imply the presence of elevated corticosteroid concentrations in the experimental situations but give no quantitative supporting data. Further, as previously emphasized, little of the previous work on the subject of phagocytosis has concerned itself with various effects as variables with time, a situation leading to a number of discrepancies in the older literature (4).

The mechanisms by which glucocorticoids bring about their multiple physiological effects are poorly understood, but seem to revolve about "permissive" effects on the permeability of cellular and intracellular membranes (5). In light of the predominantly glycolytic metabolism of peripheral blood leukocytes, it is of interest to note the work of Martin, Chaudhuri, Green, and McKinney (19), confirmed by Rauch, Loomis, Johnson, and Favour (20), demonstrating marked inhibition of glycolysis effected by in vitro incubation of intact human peripheral blood leukocytes with microgram per milliliter concentrations of certain glucocorticoids, and the lack of such effects when the cells were disrupted (20). Previous studies from this laboratory have noted the inhibition of erythrophagocytosis by in vitro incubation of leukocytes with other inhibitors of glycolysis (2), suggesting that the pharmacologic effect of glucocorticoids in suppressing phagocytosis in vivo at least by peripheral blood cells may be accomplished through an ultimate effect on the energy yielding metabolism of the leukocyte. That this is not a simple phenomenon, however, is indicated by the gross quantitative differences in the amount of glucocorticoid necessary to produce inhibition of erythrophagocytosis when incubated in vitro with the leukocyte suspension as opposed to the amounts necessary to produce inhibition when administered directly to the leukocyte donor.

A question may be raised over whether the glucocorticoid inhibition of erythrophagocytosis observed in the present studies could be explained by differences in the maturity, and hence phagocytic avidity, of the neutrophils. It would appear that the experiments in which plasma containing high levels of administered glucocorticoids inhibited cells from blood containing normal levels of glucocorticoids, and vice versa, argue against this as a major explanation for the effects demonstrated.

The experimental results obtained in this work indicate that the phenomenon of erythrophagocytosis is very responsive to the presence of glucocorticoid compounds in the circulating blood and suggest the possibility that simple modifications of these experiments might serve as excellent clinical tests of adrenal cortical responsiveness to ACTH.

\section{Summary}

Experiments were conducted to assess the effects of ACTH and certain adrenal corticosteroids 
on the in vitro phagocytic capability of human peripheral blood leukocytes for opsonized erythrocytes. In vitro addition of glucocorticoids required grossly unphysiological amounts of these substances to cause inhibition. In contrast, intravenously administered cortisol and orally administered prednisone caused sequential decrease in the erythrophagocytic capacity of leukocytes from both normal subjects and patients with chronic adrenal cortical insufficiency. Intravenously administered ACTH showed a similar effect in normal patients but yielded no effect in patients with Addison's disease. It is suggested that the techniques employed may lend themselves to clinically useful methods for assessing certain aspects of adrenal glucocorticoid metabolism.

\section{Acknowledgment}

The authors gratefully acknowledge the assistance of Dr. A. Dutton in performing the statistical analysis in this study.

\section{References}

1. Packer, J. T., R. M. Greendyke, and S. N. Swisher. Inhibition of erythrophagocytosis in vitro by corticosteroids. Trans. Ass. Amer. Phycns 1960, 73, 93.

2. Greendyke, R. M., R. E. Brierty, and S. N. Swisher. In vitro studies on erythrophagocytosis. Blood 1963, 22, 295.

3. Plager, J. E., P. Cushman, Jr., and A. E. Chase. The plasma cortisol response to ACTH in "idiopathic hirsutism." J. clin. Invest. 1961, 40, 1315.

4. Greendyke, R. M., R. E. Brierty, and S. N. Swisher. In vitro studies on erythrophagocytosis. II. Effects of incubating leukocytes with selected cell metabolites. J. Lab. clin. Med. 1964, 63, 1016.

5. Bush, I. E. Chemical and biological factors in the activity of adrenocortical steroids. Pharmacol. Rev. 1962, 14, 317.

6. Gordon, A. S., and G. F. Katsh. The relation of the adrenal cortex to the structure and phagocytic activity of the macrophagic system. Ann. N. Y. Acad. Sci. 1949, 52, 1.

7. Heller, J. H. Cortisone and phagocytosis. Endocrinology $1955,56,80$.
8. Reichard, S. M., A. Edelmann, and A. S. Gordon. Adrenal and hypophyseal influences upon the uptake of radioactive gold $\left(\mathrm{Au}^{188}\right)$ by the reticuloendothelial system. Endocrinology 1956, 59, 55.

9. Reichard, S. M., A. Edelmann, and A. S. Gordon. Endocrine influences upon the uptake of colloidal thorium by the reticuloendothelial system. J. Lab. clin. Med. 1956, 48, 431.

10. Nicol, T., and D. L. J. Bilbey. Substances depressing the phagocytic activity of the reticuloendothelial system. Nature (Lond.) 1958, 182, 606.

11. Snell, J. F. The reticuloendothelial system. I. Chemical methods of stimulation of the reticuloendothelial system. Ann. N. Y. Acad. Sci. 1960, 88, 56.

12. Crepea, S. B., G. E. Magnin, and C. J. Seastone. Effect of ACTH and cortisone on phagocytosis. Proc. Soc. exp. Biol. (N. Y.) 1951, 77, 704.

13. Moeschlin, S., W. Zurukzoglu, and J. Crabbé. Untersuchungen über den Einfluss von Cortison und ACTH auf die Phagozytose der Leukozyten und Makrophagen. Acta haemat. (Basel) 1953, 9, 277.

14. Crabbé, J. The effects of cortisone on the phagocytic activity of rabbit macrophages to staphylococci. Acta endocr. (Kbh.) 1955, 18, 251.

15. Crabbé, J. The effects of ACTH on the phagocytic activity of rabbit macrophages to staphylococci. Acta endocr. (Kbh.) 1955, 18, 256.

16. Crabbé, J. Enhancing action of small doses of cortisone on macrophage phagocytosis of staphylococci in rabbits. Acta endocr. (Kbh.) 1956, 21, 41.

17. Ludány, G., J. Rigò, J. Sós, and G. Vajda. Prednisolon-Na-succinat und die Phagozytose der Leukozyten. Experientia (Basel) 1959, 15, 463.

18. Hirsch, J. G., and A. B. Church. Adrenal steroids and infection: the effect of cortisone administration on polymorphonuclear leukocytic function and on serum opsonins and bactericidins. J. clin. Invest. 1961, 40, 794.

19. Martin, S. P., S. N. Chaudhuri, R. Green, and G. R. McKinney. The effect of adrenal steroids on aerobic lactic acid formation in human leukocytes. J. clin. Invest. 1954, 33, 358.

20. Rauch, H. C., M. E. Loomis, M. E. Johnson, and C. B. Favour. In vitro suppression of polymorphonuclear leukocyte and lymphocyte glycolysis by cortisol. Endocrinology 1961, 68, 375. 